\title{
Reconstruction of velocity profiles of two-dimensional targets by an inverse scattering procedure based on a numerical forward solver
}

\author{
Massimo Brignone, Matteo Pastorino*, Mirco Raffetto, and Andrea Randazzo \\ Department of Electrical, Electronic, Telecommunication Engineering, and Naval \\ Architecture, University of Genoa, Genoa, Italy
}

In this contribution we investigate the capabilities of inverse scattering procedures for electromagnetic problems involving axially moving cylinders, without any restriction on their number, their shapes and their profiles of relative dielectric permittivity and axial velocity. As usual, the inverse scattering problems of interest are recast as optimization problems. An Ant Colony Optimization procedure is adopted to numerically solve these problems. It exploits a proprietary finite element code for the solution of forward scattering problems involving objects in motion. When the maximum magnitude of the axial speed is limited to onehundredth of the speed of light in vacuum, it has been shown that it could be convenient to split the inverse problem into two parts (M. Pastorino, M. Raffetto, and A. Randazzo, IEEE Trans. Geosci. Remote Sens., 53, 2015, pp. 1452-1462): the first one related to the reconstruction of the geometrical and dielectric unknowns while the second part has to deal with the determination of the velocity profile. In this work we focus, in particular, on the latter one.

As a first preliminary example, we have considered the reconstruction of a velocity profile in a circular pipe, whose center is assumed to be placed in the origin of a Cartesian reference system in the transverse plane. The pipe is $10 \mathrm{~cm}$ thick, has an inner radius of $1 \mathrm{~m}$ and is made up of a lossy homogeneous material having a relative permittivity equal to $2-j$. The relative magnetic permeability of all materials involved is supposed to be equal to 1 . The pipe is hosted in a lossy medium characterized by a relative permittivity equal to $1-j 0.1$ and is illuminated by a uniform TM polarized plane wave propagating as a regressive wave along the $y$ axis. Its frequency is $100 \mathrm{MHz}$. Inside the pipe a homogeneous lossless medium is considered. It is supposed to have a relative permittivity equal to 5 in its rest frame. Its velocity profile is not constant and is given by $0.001(4-3 y) c$, being $c$ the speed of light in vacuum. The finite element forward scattering solver considers a numerical domain of radius $3 \mathrm{~m}$ and discretize it by using a mesh having 10981 nodes, 21600 triangular elements and 360 boundary edges.

In the very first reconstruction attempts, the elements have been clustered in 9 subregions and a fixed velocity value has been assigned to all elements of a given subregion. The indicated subregions are determined by dividing the domain with the following four lines $x=1 / 3$, $x=-1 / 3, y=1 / 3$ and $y=-1 / 3$. The best reconstruction of the velocity profile has required $15 \mathrm{ACO}$ iterations, with 272 calls to the finite element solver. The final mean relative error of the axial speed values was about $28 \%$, having considered as reference solution the one which is obtained by using the analytical values of the velocity field at the centers of gravity of all subregions considered. The CPU time required to complete this very first reconstruction process was about two hours on a Dell Precision T1700 (Intel Core I7-4770 processor at $3.4 \mathrm{GHz}$ ). 